University of Wollongong

Research Online

Australian Institute for Innovative Materials -

Papers

Australian Institute for Innovative Materials

$1-1-2016$

\title{
Hierarchical Nafion enhanced carbon aerogels for sensing applications
}

Bo Weng

Southwest University, bweng@uow.edu.au

Ailing Ding

Southwest University

Yuqing Liu

University of Wollongong, yl037@uowmail.edu.au

Jianglin Diao

Southwest University

Joselito M. Razal

Deakin University, jrazal@uow.edu.au

See next page for additional authors

Follow this and additional works at: https://ro.uow.edu.au/aiimpapers

Part of the Engineering Commons, and the Physical Sciences and Mathematics Commons

Research Online is the open access institutional repository for the University of Wollongong. For further information contact the UOW Library: research-pubs@uow.edu.au 


\title{
Hierarchical Nafion enhanced carbon aerogels for sensing applications
}

\begin{abstract}
This work describes the fabrication of hierarchical 3D Nafion enhanced carbon aerogels (NECAGs) for sensing applications via a fast freeze drying method. Graphene oxide, multiwalled carbon nanotubes and Nafion were mixed and extruded into liquid nitrogen followed by the removal of ice crystals by freeze drying. The addition of Nafion enhanced the mechanical strength of NECAGs and effective control of the cellular morphology and pore size was achieved. The resultant NECAGs demonstrated high strength, low density, and high specific surface area and can achieve a modulus of $20 \mathrm{kPa}$, an electrical conductivity of $140 \mathrm{~S} \mathrm{~m}-1$, and a specific capacity of $136.8 \mathrm{~F} \mathrm{~g}-1$ after reduction. Therefore, NECAG monoliths performed well as a gas sensor and as a biosensor with high sensitivity and selectivity. The remarkable sensitivity of $8.52 \times 103 \mu \mathrm{A} \mathrm{mM}-1 \mathrm{~cm}-2$ was obtained in dopamine (DA) detection, which is two orders of magnitude better than the literature reported values using graphene aerogel electrodes made from a porous $\mathrm{Ni}$ template. These outstanding properties make the NECAG a promising electrode candidate for a wide range of applications. Further in-depth investigations are being undertaken to probe the structure-property relationship of NECAG monoliths prepared under various conditions.
\end{abstract}

\section{Disciplines}

Engineering | Physical Sciences and Mathematics

\section{Publication Details}

Weng, B., Ding, A., Liu, Y., Diao, J., Razal, J. M., Lau, K., Shepherd, R. L., Li, C. \& Chen, J. (2016). Hierarchical Nafion enhanced carbon aerogels for sensing applications. Nanoscale, 8 (6), 3416-3424.

\section{Authors}

Bo Weng, Ailing Ding, Yuqing Liu, Jianglin Diao, Joselito M. Razal, King Tong Lau, Roderick L. Shepherd, Changming $\mathrm{Li}$, and Jun Chen 


\title{
Hierarchical Nafion Enhanced Carbon Aerogels for Sensing Applications
}

\author{
Bo Weng*, a Ailing Ding, ${ }^{a}$ Yuqing Liu, ${ }^{b}$ Jianglin Diao, ${ }^{a}$ Joselito Razal, ${ }^{c}$ King Tong Lau, ${ }^{d}$ Roderick \\ Shepherd, ${ }^{e}$ Changming $L i^{a}$ and Jun Chen ${ }^{* b}$
}

\begin{abstract}
This work describes the fabrication of hierarchical 3D Nafion enhanced carbon aerogels (NECAGs) for sensing applications via fast freeze dry method. Graphen Oxide, Multiwall Carbon Nanotubes and Nafion were mixed and extruded into liquid nitrogen followed by the removal of ice crystal by freeze dry. The addition of nafion enhanced the mechanical strength of NECAGs and achieves effective control of cellular morphology and pore size. The resultant NECAGs demonstrated high strength, low density, high specific surface area and can achieve modulus of $20 \mathrm{kPa}$, electrical conductivity of $140 \mathrm{~S} / \mathrm{m}$, specific capacity of $136.8 \mathrm{~F} / \mathrm{g}$ after reduction. Therefore, NECAG monoliths performed well as a gas sensor and as a biosensor with high sensitivity and selectivity. The remarkable sensitivity of $8.52 \times 10^{4} \mu \mathrm{A} / \mu \mathrm{M} . \mathrm{cm}^{2}$ was obtained in dopamine (DA) detection, which is two orders of magnitude better than literature reported values using graphene aerogels electrode made from porous Ni template. These outstanding properties make the NECAG as promising electrode candidate for a wide range of applications. Further in-depth investigations are being undertaken to probe the structure-properties of NECAG monoliths prepared under various conditions.
\end{abstract}

\section{Introduction}

Highly porous aerogels have attracted considerable attention since it was first reported by Kistler in the $1930 \mathrm{~s}^{[1]}$. The multiple functional properties of aerogels, such as high porosity, low density, ultra-lightweight, make these materials promising for a wide range of applications including chemical and biological sensing, energy storage, and catalyst platform. Early studies on aerogel synthesis have mainly focused on silica gels ${ }^{[2]}$, sol-gel materials ${ }^{[3]}$, cross-linked polymers and regenerated cellulose materials ${ }^{[4]}$. Recently, these developments have been extended to nanocarbon-based aerogels, such as carbon nanotubes and graphene, because of their outstanding mechanical and electrical properties, and high specific surface area ${ }^{[5,6]}$.

To date, three dimensional (3D) carbon aerogels (CAGs) have proven to be very attractive for many applications including supercapacitors $^{[7-9]}$ and sensors ${ }^{[10-12]}$ because of their high electrical conductivity, high porosity and high specific surface area. Given the remarkable potential of CAGs, many research groups have explored a variety of approaches to produce and characterize the applicability of CAGs for a range of applications. Bao et al. generated interconnected CAGs through hydrothermal treatment of carbohydrates (glucose, bcyclodextrin, and chitosan) and utilized them as supercapacitor electrodes. These CAG electrodes showed excellent specific capacitance $(161.6 \mathrm{~F} / \mathrm{g})$ and electrical conductivity $(1.27 \mathrm{~S} / \mathrm{m})$. It was shown that it is the $3 \mathrm{D}$ interpenetrating micro structure of CAG electrodes that has enabled fast ionic transport for electrochemical energy storage ${ }^{[7]}$. Chen et al. developed 3D macroporous CAG sensors by chemical vapor deposition (CVD) of graphene onto a porous nickel foam template. Free-standing 3D CAGs were obtained after the removal of metal platform ${ }^{[10]}$ and were used as dopamine (DA) sensors that exhibited high sensitivity $\left(619.6 \mu \mathrm{A} \mathrm{mM}^{-1} \mathrm{~cm}^{-2}\right)$. These CAG electrodes were further modified for the enzymeless detection of hydrogen peroxide $^{[12]}$ and glucose $\mathrm{e}^{[11]}$.
Freeze drying of graphene oxide (GO) dispersions can also be used to generate CAGs with high specific surface area (SSA) ${ }^{[13-}$ ${ }^{15]}$. Also multiwall carbon nanotubes (MWNTs) have been used as a common additive to improve the electrical conductivity and mechanical properties of graphene-based CAGs. Unfortunately, due to the instability and poor connections of graphene oxide sheets during freeze drying, these CAGs showed poor mechanical property, low conductivity and uncontrollable porosity ${ }^{[14]}$ even after the addition of MWCNTs.

Nafion is a commonly used ionic conductor in electrochemical applications (sensors, fuel cells etc. ${ }^{[16]}$ to enhance the electron transfer and device performance. Nafion has also been reported to form highly ordered 3D honeycomb cellular structures when freeze dried. ${ }^{[17]}$ It has also been shown to enhance the mechanical strength of aerogel materials ${ }^{[18]}$, such as cellulose aerogels.

Most CAGs made with nanocarbon materials (MWCNTs, graphene etc.) are hydrophobic which limits the utilisation of their high surface area in electrochemical sensing applications, especially in aqueous environment. The difference in surface energy between water and electrodes prevents biomolecules to access the micron pores of the CAG electrode or to come in contact with the CAG inner cell walls. This leads to limited performance of CAGs in electrochemical sensing.

In addition it has been reported that Nafion can enhance the selectivity of DA sensing. ${ }^{[22]}$ The negatively charged sulfonate group of Nafion can repulse the negatively charged AA and UA and attract positively charged DA to NECAGs.

Therefore, as a hydrophilic and ionic polymer, the introduction of Nafion into a GO/MWCNTs system can be expected to improve the hydrophilic property of CAGs to allow free entry of biomolecules into micro sized pores, enhance the selectivity in DA detection and serve as a binder that can induce the formation of highly ordered 3D honeycombed structures. It can also be expected that the resulting unique architecture can result in enhanced mechanical strength and electrochemical performance when used as electrodes for electrochemical applications. 
In the present work, we describe the facile fabrication of $3 \mathrm{D}$ CAG based on GO, MWNT and Nafion framework, referred to as Nafion-enhanced CAG, or NECAG. We show that it is the addition of Nafion that has enabled the control of cellular morphology and pore size. We demonstrate that the ultra-low density, high specific surface area, good electrical conductivity and mechanical properties of NECAG are suitable for sensing applications, such as in the enzyme-free electrochemical sensing of dopamine (DA) and in organic vapor sensing. Furthermore, preliminary studies on specific capacity (Table S2) show promising potential for use as elastic supercapacitors. It is the first time (to our knowledge) to utilise freeze dried carbon aerogel in electrochemical sensing applications as the introduction of Nafion into CAGs has improved the hydrophilic property of CAGs as well as the interaction between biomolecules and sensor electrode.

\section{Materials and Methods}

\section{Chemicals}

All chemicals utilized in this work were all purchased from sigma-Aldrich and used as received.

\section{Preparation of GO}

GO dispersions were prepared using the previously reported modified Hummers' method ${ }^{[21]}$.

\section{Preparation of CAG}

Before GO and MWCNTs were mixed at the weight ratio of 2:1 together, the two components were treated by probe sonication for $1 \mathrm{~h}$ ( $1 \mathrm{~s}$ on, $1 \mathrm{~s}$ off, $30 \%$ amplitude) to ensure good dispersion, then followed by $24 \mathrm{~h}$ continuous bath sonication after mixing. Nafion was added in the last two hours of sonication. The concentration of GO and MWCNTs were both prepared as 10 $\mathrm{mg} / \mathrm{mL}$ in water and mixed at the ratio $2: 1$ according to previous report to achieve the balance between mechanical strength and conductivity ${ }^{[14]}$. The mixtures were injected into liquid nitrogen and then freeze-dried (CHRIST ALPHA2-4 LD Plus) to prepare the NECAG monoliths. To prepare a probe electrode as in Figure 4(a), the mixtures were firstly extruded into a steel needle with a diameter of $1 \mathrm{~mm}$ and fast frozen in liquid nitrogen to form a probe shape. Pt wire can be inserted into the needle to be frozen together with NECGAs. NECAGs cylinder for sensing could be obtained after the removal of water by freeze dry under $-80^{\circ} \mathrm{C}$.

\section{Physical characterization of NECAGs}

Scanning electron microscopy (SEM) images were taken by JSM-6510LV, Japan. Thermogravimetric analysis was performed using a Q50 TGA system and Q20 DSC system (TA Instruments-Waters LLC, US). $4 \mathrm{mg}$ sample was used for the analysis. Samples were heated in nitrogen from $50{ }^{\circ} \mathrm{C}$ to $850{ }^{\circ} \mathrm{C}$ at a heating rate of $5{ }^{\circ} \mathrm{C} / \mathrm{min}$. X-Ray diffraction (XRD) measurements were performed on a Shimadzu diffractometer (XRD-7000, Tokyo, Japan) operating in reflection mode with $\mathrm{Cu}$ $\mathrm{K} \alpha$ radiation at a step size of 0.06 per second. Fourier Transform infrared spectroscopy (FTIR) spectra were recorded using a Thermo Nicolet 6700 FTIR spectrometer from 400 to $4000 \mathrm{~cm}$ 1. The specific surface area of NECAGs was obtained using a
Brunauer-Emmett-Teller apparatus (BET, Micromeritics, ASAP2020). The compressive tests were performed in an Instron (Micro Tester, 5848, Instron) using a $10 \mathrm{~N}$ load cell and strain control mode with a strain rate of $1 \mathrm{~mm} / \mathrm{min}$. The electrical conductivity of NECAGs was characterized using the set-up shown in Figure 4(a) where the resistivity (R) of NECAG monoliths was measured by a multimeter. As $\mathrm{R}=\rho \times \mathrm{L} / \mathrm{A}=$ $\rho \times 4 \mathrm{~L} / \pi \mathrm{D}^{2}$ (ohm law), thus the conductivity (S) of CAG $\mathrm{S}=1 / \rho=$ $4 \mathrm{~L} / \pi \mathrm{RD}^{2}$, where $\mathrm{L}$ is the length and $\mathrm{D}$ is the diameter of the probe as shown in Fig. S2.

\section{Gas sensing characterization of CAG}

The gas sensing ability of CAG was characterized using the settings shown in Figure S3. CAG electrodes connecting with multimeter were put in a sealed chamber to for harmful vapor detection. The resistivity will be changed when harmful vapor injected.

\section{Electrochemical characterization of CAG}

$\mathrm{CV}$ and capacitance were characterized using an CHI 660 system (Shanghai Chenhua, China). CAG electrodes served as working electrodes with a platinum mesh as counter electrode and $\mathrm{Ag} / \mathrm{AgCl}$ (in $3 \mathrm{M} \mathrm{KCl}$ ) as reference electrode. $\mathrm{CV}$ curves without DA were recorded using in $10 \mathrm{~mL} 0.1 \mathrm{M} \mathrm{NaNO} 3$ solution. $\mathrm{CV}$ and DPV curves of CAG in the presence of DA were recorded in $10 \mathrm{~mL} 0.1 \mathrm{M}$ PBS solution $(\mathrm{pH}=7)$. The specific capacity was obtained from $\mathrm{CV}$ curves according to the following equation $\mathrm{Cs}$ $(\mathrm{F} / \mathrm{g})=\mathrm{S} / \Delta \mathrm{V} . v \cdot \mathrm{m}$, where $\mathrm{S}$ is the integration area of CV Curve, $\Delta \mathrm{V}$ is the potential scan range, $\mathrm{m}$ is the mass of NECAGs electrode and $v$ is the scan rate. The electrode area was defined as $\mathrm{A}_{\text {electrode }}=\pi \mathrm{DL}+\pi \mathrm{D}^{2} / 4$, where $\mathrm{L}$ is the length and $\mathrm{D}$ is the diameter of the probe NECAG electrode.

\section{Results and Discussion}

NECAG monoliths with various shapes were prepared as shown in in Figure 1. Briefly formulations containing the GO/MWNTs/Nafion mixture were extruded into liquid nitrogen for rapid freezing. By using different injectors, NECAG can be shaped into multi-sized spheres (Figure 1 (a)), cylinders, (Figure 1 (d)), and flat sheets. These sheets can be deposited on substrates such as PVDF membrane, carbon cloth, Ni cloth, etc. (Figure 1 (g)). As these NECAG monoliths were frozen in liquid nitrogen in seconds, the original ordered structures of components in dispersion can be maintained to most extent. The removal of ice crystals from the frozen mixture by freeze drying has resulted in the formation of highly organized cellular framework (Figure 1 (f)). It is noted that without Nafion, the GO/MWNT CAG displayed discontinuous structure (Figure 2a and Figure S1). It was observed in some areas of CAGs that MWNTs were connected to adjacent GO sheets but these connections are loose and easy to break.

With the GO/MWNT ratio fixed to 2:1, NECAG with various Nafion loadings (C/Nafion $=3: 1,5: 1,10: 1, \mathrm{C}=\mathrm{GO}+\mathrm{MWCNTs}$, weight ratio) were prepared to evaluate the effect of Nafion concentration on NECAG morphology. It was observed that the pore size of the resulting NECAG vary with Nafion loading. SEM and BET characterizations of NECAG with various Nafion 
loading revealed that high Nafion concentration $(\mathrm{C} / \mathrm{Nafion}=3: 1)$ has resulted in large-sized pores $(80$ to $100 \mu \mathrm{m})$ but with low surface area. In comparison, lower Nafion loadings $(\mathrm{C} / \mathrm{Nafion}=$ 5:1 and 10:1) has resulted in NECAGs with smaller pore sizes (30 to $40 \mu \mathrm{m}$ and 10 to $20 \mu \mathrm{m}$, respectively) but with higher surface area (Table S1). It is likely that Nafion functions as binder for the GO and MWNT, which could explain the formation of continuous cellular network morphology. Therefore, as Nafion concentration increases, the Nafion that are adhered to the cell walls also thickens. As the wall thickens, small pores group together to form the large pores, and effectively decreasing the surface area. Notably, NECAGs with relatively low Nafion loading $(\mathrm{C} / \mathrm{Nafion}=10: 1)$ still revealed highly ordered and continuous cellular morphology.

Annealed NECAGs (heated at $600{ }^{\circ} \mathrm{C}$ for $5 \mathrm{~h}$ in Argon) were also investigated. High magnification SEMs show that decomposed Nafion (as dark spots in Figure S3) and MWNTs (Figure S4) are distributed on the cell walls of NECAG $(\mathrm{C} / \mathrm{Nafion}=3: 1)$, which verify that the cell walls constitute of the three components. The SSA of NECAGs increased after annealing possibly due to the decomposition of Nafion and partially removal of functional groups in GO which expose more active areas (Figure S5 (b), Table S1). Also, while NECAG retained their original porous structure after annealing (Figure S5), the porosity of samples without Nafion (i.e. CAGs) no longer appear regular. Compressive mechanical tests revealed that NECAGs demonstrated high mechanical properties. In particular, annealed NECAG with C/Nafion ratio of 3:1 revealed excellent resilience when released from $80 \%$ of compression, similar to the properties of other materials with honeycomb structure ${ }^{[6,8]}$ Shown in Figure 3(a) is NECAG with a density of $0.212 \mathrm{mg} / \mathrm{cm}^{3}$ that can achieve a modulus of $20 \mathrm{kPa}$. This strength is equivalent to sustaining 4,800 times of its own weight. This value is twice that of reported CAG made from GO and MWNT without Nafion ${ }^{[19]}$. Figure 3(a) also demonstrated that higher Nafion concentration leads to higher mechanical strength, possibly due to the reinforcing effect of thicker cell walls.

The physical and chemical properties of NECAG under various reduction conditions were investigated further to identify their suitability for sensing applications.

Thermogravimetric analysis (TGA) and differential scanning calorimetry (DSC) (Figure 3 (b)) were performed to characterize the thermal stability of NECAGs and three constituents (MWCNTs, Nafion and GO) respectively. There is no obvious weight loss in TGA curve and peaks in DSC curve of MWCNTs, illustrating MWCNTs were thermally stable from room temperature to $750^{\circ} \mathrm{C}$. The decomposition of Nafion started from $300^{\circ} \mathrm{C}$ and completed at around $500^{\circ} \mathrm{C}$, demonstrating that thermal reduction of NECAGs beyond $500{ }^{\circ} \mathrm{C}$ could eliminate the influence of Nafion in NECAGs. TGA and DSC of GO showed the reduction of GO started at around $200{ }^{\circ} \mathrm{C}$ and was maintained to the end of characterisation. Both the decomposition of Nafion and the reduction of GO could be observed in the thermal characterisation of NECAGs. The first stage of weight loss observed at around $200{ }^{\circ} \mathrm{C}$ in TGA of NECAG could be attributed to the start of GO reduction and the second stage of weight loss observed between $300^{\circ} \mathrm{C}$ and $500{ }^{\circ} \mathrm{C}$ represented the degradation of Nafion.

From FTIR spectra of NECAGs (Figure 3(c)), the absorption peak at $3250 \mathrm{~cm}^{-1}$ which corresponds to the $\mathrm{O}-\mathrm{H}$ stretching vibrations verified the existence of $-\mathrm{OH}$ group and $-\mathrm{COOH}$ group in GO. The peaks at 1725 and $1620 \mathrm{~cm}^{-1}$ are assigned to the $\mathrm{C}=\mathrm{O}$ and $\mathrm{C}=\mathrm{C}$ stretching vibrations of $-\mathrm{COOH}$ groups, respectively. The peak at $1450 \mathrm{~cm}^{-1}$ is ascribed to antisymmetric bending vibration of $\mathrm{CH}_{3}$. The existence of Nafion can also be observed from Figure 3(c). The peaks at $1200 \mathrm{~cm}^{-1}, 1130 \mathrm{~cm}^{-1}$, $1050 \mathrm{~cm}^{-1}$ and $975 \mathrm{~cm}^{-1}$ represented $-\mathrm{CF}_{2}$ groups, $-\mathrm{SO}_{3} \mathrm{H}$ groups, $\mathrm{CFRCF}_{3}$ groups and $-\mathrm{COC}$ groups in Nafion. The peak at 1540 $\mathrm{cm}^{-1}$ corresponds to the stretching vibrations of MWCNTs. The band at $650 \mathrm{~cm}^{-1}$ refers to stretching vibrations of $\mathrm{RC}=\mathrm{CRH}$.

NECAGs annealed at $600{ }^{\circ} \mathrm{C}$ for $5 \mathrm{~h}$ led to the decrease in intensities of FTIR peaks at $3250 \mathrm{~cm}^{-1}, 1725 \mathrm{~cm}^{-1}, 1620 \mathrm{~cm}^{-1}$, $1200 \mathrm{~cm}^{-1}, 1130 \mathrm{~cm}^{-1}$ and $975 \mathrm{~cm}^{-1}$, illustrating the reduction of GO and the decomposition of Nafion. This is also confirmed in the XPS C1s (Figure 3(e)) and O1s spectra (Figure S6) whereby the peak intensities for the $\mathrm{C}-\mathrm{O}(286.5 \mathrm{eV}), \mathrm{C}=\mathrm{O}(287.9 \mathrm{eV})$ in $\mathrm{C} 1 \mathrm{~S}$ and $\mathrm{O} 1 \mathrm{~S}(533 \mathrm{eV})$ in GO/MWCNTs, and the C-F $(292.1 \mathrm{eV})$ in Nafion decreased.

In contrast, when NECAGs are exposed to hydrazine vapor (at $90{ }^{\circ} \mathrm{C}$ for $2 \mathrm{hrs}$ ), similar level of GO reduction can be achieved without Nafion degradation, as can be seen in the presence of the signature peaks for Nafion in FTIR (peaks at $1200 \mathrm{~cm}^{-1}, 1130 \mathrm{~cm}^{-}$ ${ }^{1}, 1050 \mathrm{~cm}^{-1}$ and $975 \mathrm{~cm}^{-1}$ representing $\mathrm{CF}_{2}$ groups, $\mathrm{SO}_{3} \mathrm{H}$ groups, $\mathrm{CFRCF}_{3}$ groups and $\mathrm{COC}$ groups in Nafion) and XPS spectra (peak at 292.1 eV representing C-F in Nafion).

XRD results (Figure 3(f)) agreed well with what FTIR and XPS spectra demonstrated. The peak at $10.6^{\circ}$ referring to GO disappeared after the reduction of NECAGs utilising both thermal and chemical method. In addition, the peak at $19^{\circ}$ demonstrated the existence of nafion in hydrogen reduced NECAGs.

Raman spectroscopy also confirmed the reduction of GO in NECAGs after annealing and hydrazine vapor treatments (Figure 3(d)). The ratio of peak intensities for the D and $\mathrm{G}$ bands $\left(\mathrm{I}_{\mathrm{D}} / \mathrm{I}_{\mathrm{G}}\right)$ decreased in both cases, which indicated a decrease in the level of defects (i.e. the transformation of $\mathrm{sp} 3$ carbon to $\mathrm{sp} 2$ carbon upon removal of some functional groups). There is also a recovery of the second-order two phonon mode located near $2700 \mathrm{~cm}^{-1}$ illustrating the recovery of crystallinity after annealing and hydrazine vapor treatment.

The electrical conductivity of NECAG (C/Nafion =3:1) was measured using the set-up shown in Figure 4(a). NECAGs reduced at $600{ }^{\circ} \mathrm{C}$ show a conductivity of $1.4 \times 10^{2} \mathrm{~S} / \mathrm{m}$, which is two orders of magnitude higher than previously reported value of CAGs without Nafion. ${ }^{[13,19]}$ This high conductivity may be attributed to the highly ordered honeycomb structure of NECAGs. The conductivity of thermally reduced NECAGs is slightly higher than the hydrazine reduced samples, which may possibly be due to the remaining Nafion.

Due to the highly porous morphology and the high electrical conductivity of NECAGs, we tested their feasibility for vapor sensing using the set-up shown in Figure S7. We employed the thermally reduced NECAG $\left(\mathrm{C} / \mathrm{Nafion}=3: 1\right.$, annealed at $\left.600{ }^{\circ} \mathrm{C}\right)$ 
for acetone vapor sensing for its higher conductivity and to avoid possible interference caused by nafion which could conduct cations but not anions or electrons. It was observed that the resistivity of NECAG gradually increased upon the injection of acetone (Figure 4(b)), agreeing well with previously reported work on RGO and MWCNTs. ${ }^{[20]}$ The mechanism for acetone sensing by the NECAGs is partly due to the expansion of the vapor in pores which affect the connections of cell walls, leading to the increase in resistance. Another reason for the change in resistance was because the adsorption of acetone on the cell walls of NECAGs influence the electrons transfer in RGO and MWCNTs. As previously reported, residual epoxide and carboxylic groups expected in RGO and MWCNTs are electronwithdrawing and promote some holes into the conduction band. Additional p-type dopants, such as $\mathrm{NO}_{2}, \mathrm{SO}_{2}$ and $\mathrm{Cl}_{2}$, should enhance hole conduction and generate a significant decrease in resistance, and n-type dopants, such as acetone, $\mathrm{CHCl}_{3}, \mathrm{NH}_{3}$, actually causedepletion of holes from the conduction band and hence raise the resistance of our material. This sensor could also respond to $\mathrm{NH}_{3}$ and $\mathrm{CHCl}_{3}$ and showed similar trend to acetone. Acetone was chosen as a representative to demonstrate the ability of NECAGs in vapor sensing for its ease to handle. ${ }^{[20]}$ The detection limit was measured to be as low as $5 \mathrm{ppm}$, which makes NECAG a promising candidate for organic vapor sensing.

We also investigated the electrochemical properties of NECAGs. The cyclic voltammograms (CV) of NECAGs reduced under different conditions is shown in Figure S8. The current response increased dramatically after NECAGs were reduced both thermally and chemically. NECAGs annealed at $600{ }^{\circ} \mathrm{C}$ revealed a nearly square shaped $\mathrm{CV}$. The $\mathrm{CV}$ response of NECAG reduced by hydrazine vapor showed similar response, which further confirmed the effective reduction. The specific capacitance of NECAGs were calculated from CVs and shown in Table S2. Chemically reduced NECAGs demonstrated slightly higher capacitance $(136.8 \mathrm{~F} / \mathrm{g})$ than thermally reduced NECAGs $(111.3 \mathrm{~F} / \mathrm{g})$ at the scan rate of $50 \mathrm{mV} / \mathrm{s}$. This result is probably caused by the hydrophobic property of thermally reduced NECAG. This preliminary studies on specific capacity show promising potential of NECAGs for utilisation as elastic supercapacitors

The NECAGs were also evaluated as biosensing electrodes using dopamine (DA) as the model target. The hydrazine vapor reduced NECAG was chosen over the thermally reduced NECAG because of its hydrophilic property and DA selectivity(due to the presence of Nafion after reduction). Thermally reduced NECAG has also been explored in the initial stage of DA sensing study but showed poor response to DA. There is nearly no signal response observed in electrochemical detection under $10 \mu \mathrm{M}$ DA both in CV and DPV(Figure S10), illustrating the importance of Nafion in CAG system in electrochemical sensing. We assumed that is probably due to the thermally reduced NECAG is too hydrophobic to allow the entry of DA into the inner pores of the electrode due to the high surface energy between water and electrode to prevent DA to get access to the inner structure of sensor. In addition, Nafion can act as the active interface for selective DA detection. In the neutral solution (PBS solution, $\mathrm{pH}=7$ ), the negatively charged sulfonate group of
Nafion can repulse the negatively charged AA and UA and attract positively charged DA on the NECAG sensor electrode to enhance the selective detection of DA. ${ }^{[22]}$

The suitability of NECAG with various C/Nafion ratio in DA detection was optimized using $\mathrm{CV}$ in the presence of $10 \mu \mathrm{M}$ at the scan rate of 5mV/s (Figure S8(c)). NEGAG with C/Nafion ratio delivered best signal response and largest special electrochemically active surface area probably due to its large pore size and high Nafion concentration. Although the surface area of this sensor is relatively lower than NECAGs with lower nafion loading, more Nafion in the sensor made the inner structure is easier to access for DA and provided more active interface for DA sensing. Further investigation will be carried out to improve the hydrophilic property of NECAG with low Nafion loading for better sensitivity by changing reducing conditions.

Shown in Figure S8 (b) are the CVs at different scan rates of NECAG $\left(\mathrm{GO} / \mathrm{MWNTs}=2: 1, \mathrm{C}_{\mathrm{Nafion}}=25 \%\right)$ in the presence of $10 \mu \mathrm{M}$ DA. At a scan rate of $5 \mathrm{mV} / \mathrm{s}$, the oxidation and reduction peaks of DA were observed at approximately $0.18 \mathrm{~V}$ and $0.17 \mathrm{~V}$ (vs. $\mathrm{Ag} / \mathrm{AgCl}$ ), respectively. With increasing scan rate, the current produced in the redox reaction of DA increased linearly and symmetrically, which illustrate the diffusion controlled process for the DA oxidation and electron transfer procedure The anodic peak current showed a linear relationship (Ipa $\left.=0.32 v^{1 / 2}-0.48, \mathrm{R}^{2}=0.991\right)$ versus the square root of scan rate (Figure S8 (b) inset), which indicates a diffusion controlled process.

Further characterizations were carried out to further evaluate the properties of NECAG. Shown on Figures S8(c) and 4(b) are the $\mathrm{CV}$ and differential pulse voltammetry (DPV) curves of NECAG probing various DA concentration solutions. It can be seen that the oxidation peak currents from the CV and DPV curves increased linearly with DA concentration. This linear relationship is represented by Ipa $=0.06 C_{\mathrm{DA}}+4.32\left(\mathrm{R}^{2}=0.994\right.$, $\left.\mathrm{C}_{\mathrm{DA}}: 1-100 \mu \mathrm{M}\right)$ for the $\mathrm{CV}$ data, and Ipa $=0.85 \mathrm{C}_{\mathrm{DA}}+8.05\left(\mathrm{R}^{2}\right.$ $\left.=0.998, \mathrm{CDA}_{\mathrm{DA}}: 0.1-10 \mu \mathrm{M}\right)$ for the DPV data, where Ipa refers to the peak current of DPV curves and $\mathrm{C}_{\mathrm{DA}}$ refers to the concentration of DA. The sensitivity calculated from DPV data is as high as $8.52 \times 10^{4} \mu \mathrm{AMM}^{-1} \mathrm{~cm}^{-2}$, which is two orders of magnitude better than literature reported values using graphene aerogels electrode made from porous $\mathrm{Ni}$ template ${ }^{[10]}$. DPV was also carried out in the presence of $200 \mu \mathrm{M}$ uric acid (UA) and $200 \mu \mathrm{M}$ ascorbic acid (AA) mixture to identify its antiinterference ability because of the coexistence of UA, AA and DA in the human serum and the close electrochemical activity of UA/AA in the determination of DA. It can be seen that the DPV response towards DA detection remained linear under super high $\mathrm{UA}$ and AA concentration $\left(\mathrm{Ipa}=0.82 \mathrm{C}_{\mathrm{DA}}+8.69, \mathrm{R}^{2}=0.999\right.$, $\mathrm{C}_{\mathrm{DA}}: 0.1-10 \mu \mathrm{M}$, Figure $4(\mathbf{d})$ ), indicating its high selectivity. Strong peak at around $0.33 \mathrm{~V}$ represented the existence of high concentration UA and AA which cannot be separated respectively under present condition. The addition of UA and AA only slightly decreased the sensitivity to $8.22 \times 10^{4} \mu \mathrm{A} \mu \mathrm{M}^{-}$ ${ }^{1} \mathrm{~cm}^{-2}$ possibly due to their interference with DA oxidation or electron transfer on the surface of NECAG. The NECAG electrodes showed high sensitivity, good selectivity and linear 
response for DA detection at sub-micro molar concentration. We are currently investigating how to further improve the sensitivity and selectivity by evaluating NECAG electrodes with various pore size and density.

\section{Conclusion}

In conclusion, high strength and low density NECAGs with controllable pore size and morphology were successfully fabricated via an easy and one-step method. This NECAG can achieve modulus of $20 \mathrm{kPa}$, electrical conductivity of $1.4 \times 10^{2}$ $\mathrm{S} / \mathrm{m}$ and specific capacity of $136.8 \mathrm{~F} / \mathrm{g}$ after reduction. In addition, NECAG monoliths performed well as a gas sensor and as a biosensor with high sensitivity and selectivity. These outstanding properties make the NECAG as promising electrode candidate for a wide range of applications. Further in-depth investigations are being undertaken to probe the structureproperties of NECAG monoliths prepared under various conditions.

\section{Acknowledgements}

The acknowledgements come at the end of an article after the conclusions and before the notes and references. This work is financially supported by National Natural Science Fundation of China (21505108) and Technological and Developmental Grant (2015-09) from Beibei District Commission. The authors would also like to acknowledge the financial support of a PhD start-up grant SWU113111 from Southwest University (Chongqing, China) and the Fundamental Research Funds (XDJK2014C146) for the Central Universities.

\section{Notes and references}

1 S. S. Kistler, Nature 1931, 127, 741; S. Kistler, J. Phys. Chem. 1932, 36, 52

2 S. Kistler, J. Phys. Chem. 1935, 39, 79; C. A. Morris, M. L. Anderson, R. M. Stroud, C. I. Merzbacher, D. R. Rolison, Science 1999, 284, 622; D. W. Schaefer, K. D. Keefer, Phys. Rev. Lett. 1986, 56, 2199.

3 H. Tamon, H. Ishizaka, M. Mikami, M. Okazaki, Carbon 1997, 35, 791.

4 H. Jin, Y. Nishiyama, M. Wada, S. Kuga, Colloid. Surface. A 2004, 240, 63; F. Liebner, A. Potthast, T. Rosenau, E. Haimer, M. Wendland, Holzforschung 2008, 62, 129.

5 N. Stefania, C. Daniel, M. L. Ferrer, C. G. María, M Francisco del, Chem. Soc. Rev. 2012, 42, 794; K. Houria, F. B. Theodore, H. S. Joe, S. Angelique, C. A. Channing, Chem. Mater. 2006, 18, 6085.

6 W. Zhen - Yu, L. Chao, L. Hai - Wei, C. Jia - Fu, Y. Shu Hong, Angew. Chem. Int. Edit. 2013, 52, 2925.

7 C.-C. C. Ji, M.-W. W. Xu, S.-J. J. Bao, C.-J. J. Cai, Z.-J. J. Lu, H. Chai, F. Yang, H. Wei, J. Colloid. Interf. Sci. 2013, 407, 416.

8 Y. Shibing, F. Jiachun, W. Peiyi, ACS Appl. Mater. Inter. 2013, 5, 7122.

9 R. Saliger, U. Fischer, C. Herta, J. Fricke, J. Non-Cryst. Solids 1998, 225; Y. He, W. Chen, X. Li, Z. Zhang, J. Fu, C. Zhao, E. Xie, ACS Nano 2013, 7, 174.
10 X. Dong, X. Wang, L. Wang, H. Song, H. Zhang, W. Huang, P. Chen, ACS Appl. Mater. Inter. 2012, 4, 3129.

11 X.-C. Dong, H. Xu, X.-W. Wang, Y.-X. Huang, M. ChanPark, H. Zhang, L.-H. Wang, W. Huang, P. Chen, ACS nano 2012, 6, 3206.

12 F. Xi, D. Zhao, X. Wang, P. Chen, Electrochem. Commun. 2013, 26, 81 .

13 L. Qiu, J. Liu, S. Chang, Y. Wu, D. Li, Nat. Commun. 2012, 3, 1241.

14 L. V. Jemma, J. P. Avinash, M. Stephen, Adv. Mater. 2009, 21, 2180; H. Sun, Z. Xu and C. Gao, Adv. Mater. 2013, 18, 2554.

15 X. Zhang, Z. Sui, B. Xu, S. Yue, Y. Luo, W. Zhan, B. Liu, J. Mater. Chem. 2011, 21, 6494.

16 J. Zhao, Y. Yu, B. Weng, W. Zhang, A. T. Harris, A. I. Minett, Z. Yue, X.-F. Huang, J. Chen, Electrochem. Commun. 2013, 37, 32; K. Schmidt-Rohr, Q. Chen, Nat. Mater. 2007, 7, 75; P. Staiti, A. Arico, V. Baglio, F. Lufrano, E. Passalacqua, V. Antonucci, Solid State lonics 2001, 145, 101; W. Yantasee, B. Charnhattakorn, G. E. Fryxell, Y. Lin, C. Timchalk, R. S. Addleman, Anal. Chim. Acta. 2008, 620, 55.

17 L. Estevez, A. Kelarakis, Q. Gong, E. H. Da'as, E. P. Giannelis, J. Am. Chem. Soc. 2011, 133, 6122.

18 T. M. Freyman, I. V. Yannas, L. J. Gibson, Prog. Mater. Sci. 2001, 46273.

19 H. Sun, Z. Xu, C. Gao, Adv. Mater. 2013, 25, 2554.

20 D. Vineet, P. S. Sumedh , A. Srikanth, A. Srikanth Rao, J. Sujit, E. R. Kyle , P. Sungjin, S. R. Rodney, K. M. Sanjeev Angew Chem. Int. Edit. 2010, 49, 2154; J. D. Fowler, M. J. Allen, V. C. Tung, Y. Yang, R. B. Kaner and B. H. Weiller, ACS Nano 2009, 2, 301.

21 D. C. Marcano, D. V. Kosynkin, J. M. Berlin, A. Sinitskii, Z. Sun, A. Slesarev, L. B. Alemany, W. Lu, J. M. Tour, ACS nano 2010, 4, 4806.

22 P. Chen, R. Vittala, P. Niena, K. Ho, Biosensors and Bioelectronics 2009, 24, 3504; Y. Chen, T. Tan, Talanta, 1995, 42, 1181. 